\title{
BMJ Open Efficacy of therapeutic fasting and plant- based diet in patients with rheumatoid arthritis (NutriFast): study protocol for a randomised controlled clinical trial
}

\author{
Anika M Hartmann (D) , ${ }^{1}$ Melanie Dell'Oro, ${ }^{2}$ Christian S Kessler, ${ }^{1,2}$ \\ Dania Schumann, ${ }^{1}$ Nico Steckhan, ${ }^{1}$ Michael Jeitler, ${ }^{1,2}$ Jan Moritz Fischer, ${ }^{1}$ \\ Michaela Spoo, ${ }^{1,2}$ Martin A Kriegel, ${ }^{3,4}$ Jochen G Schneider, ${ }^{5,6}$ Thomas Häupl, ${ }^{7}$ \\ Farid I Kandil,, ${ }^{1,8}$ Andreas Michalsen, ${ }^{1,2}$ Daniela A Koppold-Liebscher ${ }^{1}$
}

To cite: Hartmann AM, Dell'Oro M, Kessler CS, et al. Efficacy of therapeutic fasting and plant-based diet in patients with rheumatoid arthritis (NutriFast): study protocol for a randomised controlled clinical trial. BMJ Open 2021;11:e047758. doi:10.1136/ bmjopen-2020-047758

- Prepublication history and additional online supplemental material for this paper are available online. To view these files, please visit the journal online (http://dx.doi.org/10. 1136/bmjopen-2020-047758)

Received 09 December 2020 Accepted 13 July 2021

Check for updates

(c) Author(s) (or their employer(s)) 2021. Re-use permitted under CC BY-NC. No commercial re-use. See rights and permissions. Published by BMJ.

For numbered affiliations see end of article.

Correspondence to Mrs Anika M Hartmann; anika.hartmann@charite.de

\section{ABSTRACT}

Background Previous studies have shown beneficial effects of therapeutic fasting and plant-based dietary interventions on disease activity in patients with rheumatoid arthritis (RA) for a duration of up to 1 year. To date, the effects of such interventions on the gut microbiome and on modern diagnostic markers in patients with RA have not been studied. This trial aims to investigate the clinical effects of therapeutic fasting and a plant-based diet in patients with RA, additionally considering current immunological diagnostic tools and microbiome analyses.

Methods/design This trial is an open-label, singlecentre, randomised, controlled, parallel-group clinical trial. We will randomly assign 84 patients with RA under a stable standard therapy to either (1) therapeutic fasting followed by a plant-based dietary intervention or (2) to a conventional nutritional counselling focusing on an anti-inflammatory dietary pattern according to the recommendations of the Deutsche Gesellschaft für Ernährung (German society for nutrition). Primary outcome parameter is the group difference from baseline to 12 weeks on the Health Assessment Questionnaire (HAQ). Other secondary outcomes include established clinical criteria for disease activity and treatment response in RA (Disease Activity Score 28, Simple Disease Activity Index, ACR-Response Criteria), changes in self-reported health and physical functional ability, mood, stress, quality of life, dietary behaviour via 3-day food records and a modified Food Frequency Questionnaire, body composition, changes in the gut microbiome, metabolomics and cytometric parameters. Outcomes will be assessed at baseline and day 7, after 6 weeks, 12 weeks and after 6 months.

Ethics and dissemination Ethical approval to process and analyse data, and to publish the results was obtained through the institutional review board of CharitéUniversitätsmedizin Berlin. Results of this trial will be disseminated through peer-reviewed publications and scientific presentations.

Trial registration number NCT03856190.

\section{Strengths and limitations of this study}

This is the first approach to generate a dietary therapeutic concept for patients with rheumatoid arthritis (RA) involving therapeutic fasting.

- Fasting has emerged as an effective, economic and safe therapeutic intervention for patients with rheumatoid arthritis.

- This study design can contribute to the understanding of pathomechanisms in RA, especially in the context of diet and the microbiome, through an extensive biorepository of blood and stool samples and large amounts of data to be collected.

- A limitation is the open-label dietary intervention, which may not exclude bias like non-specific treatment effects, confirmation and observer bias.

\section{BACKGROUND}

Rheumatoid arthritis (RA) is a systemic autoimmune disease, characterised by a destructive inflammation of the joints. With a prevalence of approximately $1 \%$ in Europe and the US $\mathrm{RA}$ is the most common rheumatic disorder, affecting several million people worldwide. ${ }^{1}$ Patients with RA experience painful, swollen joints which can severely impair physical function and quality of life; untreated it may lead to irreversible articular deformation and stiffness as well as to increased cardiovascular risk and mortality. ${ }^{2}$

So far, disease progression can only be stopped by permanent suppression of the inflammatory response. ${ }^{3}$ In recent years, therapeutic concepts with non-steroidal anti-inflammatory drugs and conventional disease modifying antirheumatic drugs (DMARDs) have been extended by targeted antibody therapies that can specifically block various inflammatory pathways (biological DMARDs). ${ }^{4-6}$ Although being more efficient 
in comparison to conventional DMARDs, individual treatment responses can differ widely; as a result, the optimal drug needs to be identified for each patient individually and has to be monitored for habituation and side effects. ${ }^{6}$ Nutritional medicine (NM) as well as Complementary and Integrative Medicine (CIM) alongside conventional drug therapy might be a supportive and possibly costeffective way to relieve RA symptoms, limit adverse effects of conventional drug therapies or even influence the course of the disease. ${ }^{7}$ Patients increasingly demand CIM and NM approaches; surveys indicate that up to two-thirds of patients with rheumatological conditions already use CIM, mainly for pain control. ${ }^{89}$

A complex interplay of genetic predispositions, lifestylerelated factors and environmental aspects is thought to account for the not yet fully understood pathogenesis of RA. ${ }^{10}$ Moreover, interactions with the intestinal microbiota have increasingly been discussed over the last three decades. ${ }^{11-15}$ A gut microbial imbalance (dysbiosis), characterised by the loss of metabolically and immunologically beneficial bacteria and a concomitant increase in potentially pathogenic microbes (pathobionts), is associated with several chronic inflammatory syndromes. ${ }^{16-22}$ In the case of RA, a predominance of Prevotella species in the intestinal microbiota has been associated with earlystage patients with RA in previous studies. ${ }^{23-25}$ Recent translational publications suggest that such an altered microbiota might be associated with the formation of RA-specific autoantibodies, that is, anticitrullinated protein antibodies (ACPAs), possibly triggering autoinflammatory disease in predisposed individuals. ${ }^{26-29}$

Nutrition is a pivotal variable in shaping the gut microbiota composition and function. It influences the complex host-microbiota cross-talk and hence affects host metabolism and the immune system in a multifaceted fashion; thus, changes in dietary regimens may have either beneficial or detrimental consequences for the gut microbiota and thus for overall health. ${ }^{11}{ }^{30}$ This also applies to caloric restriction or fasting, an element which can be implemented in different types of diets. ${ }^{31} 32$

In the past few decades, mounting experimental and translational evidence regarding the biological fundamentals of caloric restriction and different fasting regimes, such as periodic and intermittent fasting, has evolved. It encompasses a broad spectrum of cellular and molecular mechanisms affecting health and disease processes; fasting not only involves ketogenesis to fuel cellular energy production but also elicits a coordinated adaptive stress response: signalling pathways are activated, which bolster mitochondrial health, DNA repair and autophagy. Fasting also seems to enhance immune functions by downregulating proinflammatory cytokine expression. ${ }^{33-36}$ These well-orchestrated processes may hold promising therapeutic options for a variety of fields in medicine, including autoimmune diseases such as RA. $^{35}$ 37-40

By contrast, robust clinical evidence on the therapeutic effects of dietary and fasting interventions in patients wih
RA has been sparse; in 2009 a Cochrane review concluded 'uncertain effects' of specific dietary regimens on RA due to a lack of sufficient data. ${ }^{41}$ Currently, a growing body of literature reports on clinical improvement through plantbased nutrition and fasting in inflammatory arthritis. ${ }^{42-45}$

In early clinical trials, modified fasting (up to $500 \mathrm{kcal}$ energy intake per day) for 7-10 days followed by plantbased diet showed positive effects such as decreased morning stiffness, reduced pain and increased function in RA patients for up to 1 year. ${ }^{22}{ }^{46}$ For this reason, it is already in regular use by a number of clinical departments in Europe for the integrative treatment of RA. ${ }^{47}$ Kjeldsen-Kragh et $a t^{22}$ and Sköldstam et $a t^{48} 49$ were able to demonstrate the effectiveness of such an approach in clinical studies, of which occurred two in a randomised setting. ${ }^{46}$

Data from several clinical trials have already suggested that therapeutic fasting produces anti-inflammatory effects. ${ }^{21} 224750$ However, in the period following fasting interventions, inflammation and symptoms frequently reoccur. Previous studies have demonstrated that this process can be delayed by the implementation of specific diets or food items. ${ }^{46} 51$ However, until now no standardised recommendations for long-term stabilisation of the positive fasting effects have been developed.

In what follows, we present the protocol of a randomised, controlled clinical trial comparing an experimental antirheumatic fasting and nutrition protocol to a conventional guideline-based anti-inflammatory diet.

We hypothesise that the experimental protocol will improve RA symptoms in the mid-term and long term. In an additional experimental context, we will investigate changes (1) in metabolism and (2) in the microbiome. We further hypothesise that the anti-inflammatory effects of fasting and plant-based diets are related to changes in the composition of the individual gut microbiota.

Thereby, this trial aims to contribute to an improved understanding of underlying pathophysiological processes and to extend non-pharmacological therapeutic options for patients with RA.

\section{METHODS AND ANALYSIS}

\section{Study design}

In this prospective, open-label, clinical, randomised, controlled trial, we intend to recruit and randomise 84 participants between 18 and 70 years of age, diagnosed with RA, into two groups (figure 1). This protocol meets the standard recommendations and guidelines for randomised clinical trials. ${ }^{53}$

\section{Patient and public involvement}

Patients are not involved in the design, or conduct, or reporting, or dissemination plans of our research, but they are a key element in the choice of our outcome measures. Both the primary and several secondary endpoints consist of patient-reported outcomes (PROs). Furthermore, we provide basic educational material to promote long-term 




Figure 1 Study design. RA, rheumatoid arthritis; TRE, time restricted eating.

health literacy (online supplemental file 1) and assess regularly the burden of the trial interventions on participants. Once the trial has been published, we intend to inform participants of the results through a newsletter suitable for a non-specialist audience.

\section{Recruitment and randomisation}

Participants are recruited using three sources (figure 2): (1) by direct referral from either physicians at the Immanuel Hospital Berlin and the Charité-Universitätsmedizin Berlin, Department of Rheumatology and Clinical Immunology Berlin or (2) by direct referral from office-based rheumatologists and (3) by non-personal advertising strategies (eg, flyers, social media).

Eligibility criteria are designed to target patients with RA who are sufficiently healthy to participate safely in the interventional fasting trial-arm (table 1). Participants meeting all inclusion and no exclusion criteria are randomly assigned to one of the two treatment arms, using a 1:1 ratio. An independent research team member, outside of the project, has generated the randomisation list using blockrand library (V.1.4) with a randomised variable block approach within the statistical computing language $\mathrm{R}$ (V.3.5). The computer-generated randomisation allocation sequence has been consecutively numbered, sealed in opaque envelopes and concealed from the study personnel responsible for conducting the studies. Participants are allocated after written informed consent (online supplemental file 1 and successful screening by the responsible study physician.

Discontinuation and adherence criteria are displayed in table 2. Missed consultation appointments can either be made up in the following groups or the omitted topic must be worked on at home. Food records measure adherence to the prescribed intervention (see outcome parameters).

\section{Study settings}

This is a single-centre trial: screening visits, blood collection and dietary counselling are conducted at the Charité-Universitätsmedizin Berlin, Department of Internal and Complementary Medicine in Berlin, Germany, located at the Department of Internal and Integrative Medicine, Immanuel Hospital Berlin, Germany.

\section{Interventions}

Dietary counselling for both trial arms is carried out in small groups of up to 10 persons within 3 individual and 9 group sessions over 3 months (figure 2 ). The entire intervention takes place in an outpatient setting.

Both groups begin with a 7-day intensive course (corresponds to a fasting week in the intervention group) with daily group coaching sessions of 2 hours each and one individual coaching of $60 \mathrm{~min}$ per participant. Afterwards, participants receive one group coaching of 1.5 hours and $30 \mathrm{~min}$ of individual phone coaching in the second Pre-Intervention $1^{\text {st }}$ month $2^{\text {nd }}$ month $3^{\text {rd }}$ month

Figure 2 Schedule for both interventions.



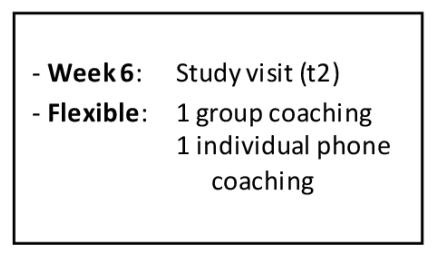

- Week 9: 3-day food record study visit ( $\mathrm{t} 3$ )

- Flexible: 1 group coaching 1 individual phone coaching 
Table 1 Eligibility criteria

\begin{tabular}{ll}
\hline Inclusion criteria & Exclusion criteria \\
\hline Prediagnosed RA* & Diagnosed gout or septic arthritis \\
Age 18-70 years & Known psychiatric disorders limiting an understanding of the \\
Ability to fully understand the trial concept and & protocol (unable to give consent) \\
written informed consent to participate & Pregnancy or breast feeding \\
Willingness to accept randomisation, to undergo & BMl $<18.5 \mathrm{~kg} / \mathrm{m}^{2}$ \\
testing and intervention procedures, and to deliver & Eating disorder (bulimia, anorexia nervosa) within the past 5 years \\
stool, blood and urine samples for testing & Severe internal diseases (eg, renal insufficiency with creatinine \\
& $>2$ mg/dL) \\
& Participation in another study \\
& Currently practising or having practised plant-based diet or fasting \\
& within the previous 6 months to enrolment \\
& Implementation of a rheumatological therapy with biologicals or \\
& biosimilars within the last 8 weeks prior to enrolment \\
& Absence of email address and internet access
\end{tabular}

*Prediagnosed by a medical specialist.

$\mathrm{BMI}$, body mass index; RA, rheumatoid arthritis.

month and third month of the study. Additionally, a cooking lesson of 3 hours is hosted in the second month. Due to the spread of COVID-19, the study protocol is being changed to maintain social distancing measures. Face-to-face consultations are carried out online using secured web-based video conferences. The cooking course is replaced by a tasting with max. 5 participants respecting hygiene measures (preparation by a single person wearing gloves, listing of the participants, distance rule, mouth covering in between tasting).

No changes to existing drug therapies are made in the course of the trial, unless undertaken by the treating rheumatologist.

\section{Fasting and plant-based dietary counselling}

The experimental intervention group undergoes a 7-day therapeutic fasting regime (2 days of light plant-based diet, 7 days of fasting, 3 days of light plant-based diet), and a following period of 11 weeks with a specific plant-based normocaloric nutrition.

During the first 2 days of light plant-based before fasting, the subjects are given a low-calorie (approx. 1200 kcal) diet with reduced intake of salt, fat and protein (online supplemental file 2). During the following fasting period, participants can consume unlimited amounts of water or herbal tea (no black or green tea, no caffeine, no alcohol), $2 \times 150 \mathrm{~mL}$ of vegetable juice low in sugar and $250 \mathrm{~mL}$ of light vegetable broth with a maximum total daily energy intake of $1.255 \mathrm{~kJ}$ (300 kcal). Participants are encouraged to drink at least 2.5 litres of fluid daily (online supplemental file 2).

In the event that a participant regularly takes medication for other diseases, his/her medication may have to be adjusted during the fasting days. Fasting is known to lower blood pressure, affect electrolyte balance and blood sugar levels. It may also prolong bleeding time and trigger migraine attacks. ${ }^{54-56}$ For this reason, diuretic, antihypertensive and antidiabetic drugs as well as coumarins are adapted by the responsible study physician (online supplemental file 3 ).

Fasting is followed by 3 days of light plant-based diet with successive reintroduction of plant-based solid food. Afterwards, a specific plant-based normocaloric diet is to be resumed. The latter is enriched with kitchen herbs and kitchen spices known for their anti-inflammatory potential. The recommended diet is also high in prebiotics and integrates the concept of time restricted eating (TRE), with 16 hours of fasting overnight for at least 6 days per week.

\section{Standard dietary counselling}

The control group receives a wholefood diet considered to be fundamentally health-promoting according to the current recommendations of the Deutsche Gesellschaft für Ernährung (DGE, German society for nutrition; online supplemental file 4$).{ }^{57}$

The omega-6-fatty acid arachidonic acid (AA), which is particularly found in animal derived protein, has been

Table 2 Discontinuation and adherence criteria

\begin{tabular}{ll}
\hline Discontinuation criteria & Adherence criteria \\
\hline Withdrawal of consent & $\geq 2$ of 3 individual consultations \\
- Medical reasons for terminating the intervention & $\geq 9$ of 11 group consultations \\
& $>1$ cooking course \\
\hline
\end{tabular}


suggested to play a role in RA as it leads to the formation of inflammation-promoting messenger substances. Therefore, a reduction of the former is thought to have an anti-inflammatory effect. ${ }^{58} 59$ Thus the focus of the control intervention is a lower intake of this substance, meaning a maximum of 2 portions per week of foods such pork lard, liver, and egg yolk. If consumption of dairy products cannot be avoided, low-fat dairy products should be preferred. High amounts of salt, sugar and alcohol should be avoided (online supplemental file 4).

The three main omega-3 fatty acids alpha-linolenic acid (ALA), eicosapentaenoic acid (EPA) and docosahexaenoic acid (DHA) have been described to have antiinflammatory effects. ${ }^{60} 61$

An enzymatic desaturation step of variable efficiency $(1 \%-5 \%)$, is required to convert dietary ALA to EPA and DHA. ${ }^{62}$ Acting as competitive inhibitors for AA, the intake of these long-chain omega-3 fatty acids in therapeutic doses of $3 \mathrm{~g}$ /day inhibits the production of proinflammatory eicosanoids.

ALA is mainly found in oilseeds such as canola, flaxseed, soybean, chia as well as walnut. DHA and EPA are found in fish like mackerel, salmon and herring. In accordance with the current recommendation of the DGE, an optimal ratio of 1:5 omega-3 to omega- 6 FA is considered. The recommended daily dose of $250 \mathrm{mg}$ EPA and DHA can be met with two servings per week of the above fish species; that of ALA using $20 \mathrm{~g}$ of walnuts, $10 \mathrm{~g}$ of linseed or $15 \mathrm{~mL}$ of either rapeseed or linseed oil per day.

An adequate supply of antioxidants, vitamins, minerals and secondary plant compounds can be achieved with five portions of fruit and vegetables daily.

\section{Adverse events}

There are no major risks expected for participants in this trial. Fasting without underlying conditions is considered safe and there are no reports of major risks whatsoever. ${ }^{63}$ Minor adverse effects of fasting therapy are described as initial headaches (coffee withdrawal, etc), mild circulatory reactions, feelings of hunger and increased sensitivity to cold. These side effects are known, occur mostly during the first 3 days of fasting and are self-limiting. ${ }^{6364}$

Occurring adverse events are recorded at each clinical visit using open questions. Adverse events are also documented at any time between visits whenever a participant communicates such an event to study personnel. Serious adverse events are announced to the study coordinator and the principal investigator within 24 hours of their report.

\section{Outcome parameters}

The primary endpoint is the group difference of the Health Assessment Questionnaire (HAQ) after 12 weeks compared with baseline. Target parameters are surveyed at baseline, on day 7 , after 6 and 12 weeks and on follow-up after 6 months (table 3 ).

Additional secondary outcomes are standard RA assessment tools (Disease Activity Score 28, DAS28;
ACR-Response-Criteria; Simple Disease Activity Index) and questionnaires for functional ability (Funktionsfragebogen Hannover), mood (Profile of Mood States), stress (Cohen's Perceived Stress Scale-10), quality of life (WHO-5) and subjective strength of the main complaint on the Visual Analogue Scale. Both groups keep a pain diary also recording pain medication intake. Occupational stress, domestic stress, interpersonal conflicts, digestion, menstruation, adherence on diet and extraordinary events are documented by means of a diary containing verbal numerical rating scale scores.

For safety monitoring, body weight and composition, food intake and vital signs are surveyed at regular intervals as well as urinalysis and a clinical standard laboratory, including blood count, liver and kidney values, vitamin $\mathrm{B}_{12}$, erythrocyte sedimentation rate and $\mathrm{CrP}$ (Biospecimen and data collection). ACPA and rheumatoid factor (IgM) are collected at baseline to complete the rheumatological assessment.

Cytometric parameters indicating changes in cell activation are determined to analyse the immunological effects of the intervention: detailed changes in subpopulations of immunological importance for inflammation (eg, monocytes) are quantified by transcriptome analysis of immune cells (microarray /RNAseq). A preliminary study by the same research group identified inflammatory profiles of individual foods as well as molecular markers of disease activity in RA, whose diagnostic value has been tested and interpreted under the influence of fasting. ${ }^{65}$ In this trial, these markers are being clinically evaluated.

Moreover, polar metabolic plasma metabolites are of interest and are analysed using a GC/MS metabolomics platform. Stool samples are collected in a subgroup of participants for 16s-18s rRNA sequencing.

Individual dietary habits are surveyed in week $-1,4$ and 9. Each measurement consists of a weighed 3-day food record (two weekdays and one weekend day) where all consumed food and drinks are recorded by the participants themselves. Exact time, mood and location during food intake are also to be recorded.

All surveys and endpoints shall be collected and assessed by trained and experienced staff. Participants are informed about any abnormal findings and referred to their general practitioner for further treatment as soon as such findings may occur.

\section{Biospecimen and data collection \\ Clinical data}

Clinical visits are carried out by study personnel at the Institute of Social Medicine, Epidemiology and Health Economics, Charité-Universitätsmedizin Berlin, Germany. Study participants are called in with an empty stomach between 8:00 am and 2:00 pm.

\section{Diet and lifestyle assessment}

Standardised electronic questionnaires (LimeSurvey V.3) are used to assess baseline data and deviations over the 
Open access

Table 3 SPIRIT flow diagram of the NutriFast trial



$\mathrm{AE}$, adverse event; SAE, serious adverse event; TRE, time restricted eating. 
study period. Questionnaires are sent out online via email and completed on the day of the respective visit (table 3).

\section{Adherence}

Daily group consultations with a nutritionist support adherence to the initial fasting week. The study team examines 3-day food records in week $-1,4$ and 9 as well as disseminates electronic questionnaires at the time of study visits to assess applicability (table 2). Food records are analysed for nutrient content using NutriGuide software. This software's database is based on the German Federal Food Code (BLS) of the German Federal Institute for the Protection of Consumer Health and Veterinary Medicine (BGVV) and provides comprehensive information about the composition/ingredients of more than 15000 foods. Participants are further asked to provide information on their lifestyle factors (including physical activity, nicotine, alcohol and illicit drug consumption) and complete a modified Food Frequency Questionnaire (FFQ) which queries the consumption frequency of certain spices and herbs at all three food recording points. The FFQ has been generated for the purpose of this trial on the basis of the clinically used FFQ of our department for integrative medicine. Its focus lies on nutrition, fasting and chronobiology.

\section{Anthropometric measurements and body composition}

Weight and height are determined at baseline, 6 weeks and 12 weeks with regularly calibrated personal scales (seca, model 920). Body composition is assessed using a bioelectrical impedance analyser (Data Input, Nutriguard $\mathrm{M} / \mathrm{S}$, model BIA-2000S) and the corresponding software by the same supplier (Data Input, NutriPlus 6.0) at each clinical evaluation.

\section{Blood samples}

Blood samples are taken at each clinical visit (baseline, 7 days, 6 weeks, 12 weeks). These are either transported directly to the study laboratory or centrifuged and frozen. Serum and EDTA samples are centrifuged at $2800 \mathrm{rpm}$ for 10 min to obtain serum and plasma, aliquoted (minimum $200 \mu \mathrm{L}$ per aliquot) and then stored at $-80^{\circ} \mathrm{C}$ for subsequent analysis. Samples from this study are only used for the ongoing process. Should further research with biological samples obtained be planned in the future, renewed informed consent will be sought.

\section{Metabolomics}

Blood samples are collected as dried blood spots and metabolites will be extracted using an established protocol $^{66}$ before assessment via GC/MS. The raw GC/ MS data will be analysed using the MetaboliteDetector software (http://metabolitedetector.tu-bs.de) to extract pure mass spectra and semi-quantitative values for all detected compounds in a non-targeted approach. ${ }^{67}$ If possible, the software will perform an identification of compounds present in a reference library. Finally, statistical analyses of the results will yield metabolites significantly affected by our treatments.

\section{Urine and stool samples}

Midstream urine samples are collected at each clinical visit (baseline, 7 days, 6 weeks, 12 weeks) and analysed immediately using semiquantitative urine dipsticks. Stool samples are collected in a subgroup of participants $(n=20)$ to the indicated timepoints (table 3). Longitudinal changes in the composition of the intestinal microbiome will be assessed using 16S rDNA gene-based next-generation sequencing on the Illumina MiSeq platform. The V4 region of $16 \mathrm{~S}$ rDNA will be amplified by PCR, normalised, pooled and sequenced with the Illumina MiSeq $2 \times 250 \mathrm{bp}$ paired end as described. ${ }^{68} 69$ Analysis of the $16 \mathrm{~S}$ sequencing results will be performed as described. ${ }^{70}$

\section{Statistical analysis}

Sample size and power calculation

Based on the estimated yearly turnover of the associated hospitals and clinics in Berlin, 84 patients were enclosed in this pilot study. Under the standard assumptions of a significance level of alpha $=0.05$, a beta $=0.20$ (corresponding to a power of $80 \%$ ) and a repeated analysis of variance design with a hypothesis only for the interaction term, a nonsphericity correction of epsilon $=1$, an assumed correlation among repeated measures of 0.5 , and a drop-out rate of $10 \%$, the number is sufficient to discover all effects with effect sizes of $\mathrm{f}=0.20 \quad(\mathrm{~d}=0.40)$ or higher, and thus all medium and large effects. The number is thus apt to decide whether the effect is larger or smaller than the critical effect size of $d=0.5$, indicating whether the treatment effect is to be termed clinically important.

\section{Blinding}

This is an open-label randomised controlled trial. Complete blinding of both the participants and the nutrionist to the treatments in this study is not possible due to obvious differences between the intervention arms.

\section{Data management}

Each participant receives his/her individual study ID (pseudonym) when enrolling in the study. In order to guarantee the confidentiality of participant's personal data this pseudonym is used for all data documentation. Initially, data are collected in source documents and transferred to analogue case report forms. Data sheets relating the participant's study ID to the person's contact details are stored in a locked cabinet in a locked office to which only members of the research team have access. Later, data are digitised and stored in a central database at Charité-Universitätsmedizin Berlin, Germany. Accuracy of the data entry is to be improved by double-checking the entries for expected scope and correct format. Participant files and other source data (including copies of protocols, questionnaires, original reports of test results, informed consent records and other documents concerning the conduct of the study) will be kept for at least 10 years after completion of the study. Any modifications to the 
current study protocol will be communicated to the institutional review board, all study participants and the study investigator.

\section{Data monitoring}

Given the minimal risks of the dietary interventions, this trial is monitored on a regular basis by the protocol team, without the use of a formal data monitoring committee.

The principal investigator, in close cooperation with the study coordinator and protocol team, may take the decision to discontinue the study. Such decision may be based either on adverse or serious adverse events attributed to the study intervention or for the reasons mentioned in the section 'Exclusion criteria' or because not enough participants could be recruited. Further details about its operating procedures can be obtained by contacting the corresponding author via email. The protocol team and the principal investigators meet every 3-6 months to monitor, review and discuss the study progress and trial related issues.

There is no endpoint assessment committee and no study steering committee. There are no stakeholder and public involvement groups involved.

\section{Data analysis}

The primary endpoint is the group difference in the HAQ after 12 weeks. Baseline variables with prognostic and demographic relevance, as well as the totality of the target criteria, are described accordingly and compared by Student's t-test. Statistical variables included are, in addition to absolute and relative frequency, the number of valid values, the formation of the SD, minimum, maximum, median and mean value (depending on the numerical scale used). For the protocol calculation of a primary endpoint, a repeated variance analysis is performed.

Based on prior publications, no detrimental effects of the study intervention are expected that would result in a premature termination of the study. ${ }^{6364}$ Hence, we do not plan an interim analysis. For an intention to treat analysis, we intend to replace missing values by multiple imputation using the amelia II package. ${ }^{71}$

Due to the consultation methods initiated to meet the restrictions associated with COVID-19, we introduced the additional covariate of 'rate of visits/consultations done only virtually' (ie, online video conferences or telephone calls). These additional tests will be done as a sensitivity analysis, and are therefore separate from the analysis of the primary outcome.

\section{Follow-up and evaluation of data}

In order to investigate the feasibility and the long-term effects of a dietary change after the study, we conduct a follow-up of all participants who have completed the study after 6 months. Electronic questionnaires are used to collect follow-up outcomes including HAQ, health questionnaires and questionnaires on function and diet/ lifestyle (table 3).

\section{Ethics and dissemination}

The trial, including participant information and informed consent, has been approved by the institutional review board of Charité-Universitätsmedizin Berlin and is conducted in accordance with the Declaration of Helsinki in its currently valid version, the guidelines of the International Conference on Harmonisation of Good Clinical Practice and the applicable German laws. An amendment for adaptations to our trial due to the COVID-19 pandemic was approved by the same ethics committee. Written informed consent is obtained from all participants preceding study entry by the responsible study physician (online supplemental file 1). We only ask for consent to use data and samples for the research subject described in this protocol, and do not intend to use participant data or biological samples in further future studies.

Results will be presented at national and international conferences, published in peer-reviewed journals and disseminated to rheumatologists and medical laymen. We will follow the official eligibility guidelines for authorship in all publications and do not intend to use professional writers.

\section{DISCUSSION}

To the authors' knowledge, this is the first approach to generate a dietary therapeutic concept for patients with RA involving therapeutic fasting. The study aims to provide additional evidence on promising existing laboratory research and clinical data on this dietary approach in patients with RA.

Previous nutritional recommendations, such as a gluten-free or Mediterranean diet, have remained rather experimental therapeutic approaches due to insufficient data regarding their mechanisms and clinical effectiveness. ${ }^{72-74}$ Likewise, a recently published Swedish trial of an anti-inflammatory, primarily pesco-vegetarian, diet in rheumatoid arthritis has shown potential efficacy but lacked significant clinical relevance regarding the chosen endpoint (DAS28-ESR). ${ }^{75}$ In this study, we aim to fill the the knowledge and quality gap on both preclinical and clinical sides of fasting and a plant-based diet in RA.

A meta-analysis by Genel et $a l^{76}$ suggests health benefits of a low-inflammatory diet for adults with arthritis (calorie-reduced regimens or fasting excluded). However, the quality of existing clinical trials is questionable-what is missing are professionally led nutritional interventions and a combined evaluation of laboratory and PROs. This structural aspect is reflected in the selection of certified nutritionists in this study design, together with the wideranging chosen endpoints. In addition, recent epidemiological studies on risk for RA under Mediterranean diet appear controversial and call for a specification into seropositive and seronegative RA, ${ }^{77} 78$ which will be implemented in the statistical analysis of the results of this study.

As far as mechanisms of action are concerned, caloric restrictions harbour potential immunological consequences for patients with RA. As Häupl et $a l^{65}$ have recently 
suggested, there is an enhanced turnover of monocytes with accelerated monocytopoiesis to be seen in RA; the prematurely released monocytes from the bone marrow migrate into, then inflamed, joints. ${ }^{79}$ Fasting in turn reduces the number and activity of circulating inflammatory monocytes in healthy humans and mice, without compromising the acute inflammatory response to infectious agents. ${ }^{80}$ The influence of fasting on the specific activity of monocytes in humans with RA shall now be the subject of this study.

Moreover, the study group of Jordan et $a l^{80}$ revealed that metabolic activity and gene expression patterns predicting improvement of chronic inflammatory and autoimmune disorders, such as RA and multiple sclerosis, are modifiable by fasting in mice. Using GC/MS metabolomics, this study shall point out the metabolic consequences of fasting in human subjects with RA as an example of chronic inflammatory conditions.

Growing evidence endorses the influence of diet on the imbalanced immune system in RA, which is also characterised by an increased number of proinflammatory T-helper cells 17 (Th17) and hence by a decreased Th1/Th17 ratio compared with healthy subjects. ${ }^{81}$ Protective regulatory T-cells (Treg) on the other hand tend to malfunction. ${ }^{81} \mathrm{~A}$ recent study showed that 28-day high-fibre supplementation induces an uplift in circulating Treg, favourable Th1/Th17 ratios and improvement in symptoms in RA patients. ${ }^{82}$ Interestingly, a ketogenic diet induces similar effects: not only does it result in alteration of the human and murine gut microbiota, but also reduces the levels of intestinal Th17 cells after human microbiome transplantations into germ-free mice. ${ }^{83}$ This translational study suggests that Th17 cell-promoting bifidobacteria may be suppressed not only by a ketogenic diet, but also fasting. Prevotella copri, implicated in the pathogenesis of RA, was also shown to induce Th17 cells in an arthritis model and might therefore be suppressed during fasting and ketosis. ${ }^{23}$ As ketone bodies directly inhibit bacterial growth,$^{83}$ other taxa may be affected as well. According to Ang et al, these should include Lactobacillus species that were sensitive not only to a ketogenic diet, but also to ketone ester supplementation in mice. Some Lactobacillus species have been linked to translocation to internal organs in lupus-prone models; a mechanism which may be also involved in the pathogenesis of RA that is characterised by a dysfunctional gut barrier. ${ }^{84} 85$ Lactobacillus also grows out in collagen-induced arthritis that is alleviated by another dietary intervention with resistant starch. ${ }^{86}$ One can thus speculate that these potentially disease-promoting taxa may be repressed in their niche within the gastrointestinal tract by the fasting protocol applied to patients with RA in this study. Similarly, the gut barrier dysfunction may improve by this intervention, being comparable to a high-fibre diet that was shown to ameliorate this aspect by increasing the mucus layer in the gastrointestinal tract and preventing from gut commensals to translocate to secondary lymphoid organs. ${ }^{8487}$

Aside from fasting, a plant-based diet also seems to result in a significant shift in the microbiota in some studies. ${ }^{88}{ }^{89}$ However, a recent review of Trefflich et $a \ell^{00}$ could not identify a consistent association between a vegan or vegetarian diet and a specific microbiota composition compared with omnivores. Eventually, the interplay of diet, microbiota and host physiology may be unique and person-specific, eliciting individual responses to dietary inputs. ${ }^{91}$ Therefore, future microbiome study designs should consider individual microbiome stability and evaluate such data rather on the basis of longitudinal intraindividual basis than on interindividual comparison. ${ }^{92}$ We will adopt this approach in the evaluation and interpretation of gut microbiota composition over the individual course of the study intervention.

Given the epidemiological and preclinical data on the one hand and the lack of consistent clinical data on the other, this trial has the potential to generate substantial data on the mechansims as well as on the efficacy of fasting and diet. It aims to target key issues on nutrition and microbiota research and its translation into clinical RA contexts.

A major strength of the study design is the comprehensive amount of data to be collected as well as a sizeable biorepository of blood and stool samples to be generated. Laboratory tests on inflammation and metabolic processes may contribute to further illuminate the mechanism of fasting in RA. Blood-based biomarkers will enable us to identify subgroups of RA patients that benefit to varying extents from our dietary intervention. The longitudinal collection of samples for microbiome analysis will provide further valuable information on the relationship between the microbiota and inflammatory activity in RA patients. Linkage to extensive clinical, dietary and lifestyle data will us provide further information to perform secondary epidemiological analyses to generate new hypotheses for testing in future studies.

Another strength is the avoidance of performance bias. We designed the trial in such a way that both intervention arms receive the same amount of coaching and medical visits, so that no systematic distortion of the study results should occur from this.

In addition, the experimental intervention presented, with the support of nutritional experts, is realistically feasible. The short coaching intervals in the first week of intervention as well as the outpatient study design is more likely to be accepted and put into practice by patients than regular dietary consultations that have to be planned in the long term. It is cost-effective and- in view of the recent research on the positive effects of plantbased foods ${ }^{429394}$ or the adverse effects of animal products $^{95}$ - has an overall health benefit most likely without serious side effects, if communicated by a professional nutritionist.

However, there are some limitations to our study design. First, the intervention of a plant-based diet 
itself includes a reduced intake in AA as the recommendations of the control interventions do. The latter, similar to other studied dietary forms, has indicated trends toward clinical improvement of RA in other clinical trials, although without reaching statistical significance. ${ }^{52} 75$ A plant-based diet though represents not only a reduced, but almost negligible consumption of AA. Nevertheless, this aspect might influence our resulting outcomes. Second, our dietary intervention is not blinded. The study, therefore, may be prone to bias like non-specific treatment effects, confirmation and observer bias. This is a general problem among studies aiming to investigate comprehensive dietary interventions and must be taken into account when interpreting results. From past studies in our facility, it is known that many patients apply for studies with a clear preference for one specific intervention. This expectation alone can lead to a change in behaviour and can bias the outcome. Currently, we expect people to favour the fasting-intervention. Patients who are assigned to the control group may not want to participate all together, thus distorting the drop-out rate. ${ }^{96} \mathrm{On}$ the other hand, rater and subject-expectation bias can increase placebo response and affect study outcome. ${ }^{97}$ We try to evaluate the degree of this effect by assessing both the rater's and the participant's expectation for both intervention arms before randomisation.

In summary, fasting and a subsequent specific diet may have the potential to provide a safe and costeffective complementary treatment option for RA. A better understanding of the underlying mechanisms could also be applicable to other rheumatic diseases. We hope this study will help bridge the gap between promising preclinical data and the lack of clinical data.

\section{Trial status}

The NutriFast trial is active with patient recruitment. Recruitment started May 2019 and will be approximately finished in the first quarter of 2021.

\section{Author affiliations}

${ }^{1}$ Institute of Social Medicine, Epidemiology and Health Economics, Charité Universitätsmedizin Berlin, corporate member of Freie Universität Berlin and Humboldt-Universität zu Berlin, Berlin, Germany

${ }^{2}$ Department of Internal and Integrative Medicine, Immanuel Hospital BerlinWannsee Branch, Berlin, Germany

${ }^{3}$ Institute for Musculoskeletal Medicine, Department of Translational Rheumatology and Immunology, University of Münster, Münster, Germany

${ }^{4}$ Department of Immunobiology, Yale University School of Medicine, New Haven, Connecticut, USA

${ }^{5}$ Department of Internal Medicine II, Universitätsklinikum des Saarlandes und Medizinische Fakultät der Universität des Saarlandes, Homburg, Germany

${ }^{6}$ Luxembourg Centre for Systems Biomedicine (LCSB), University of Luxembourg, Luxembourg, Luxembourg

${ }^{7}$ Department of Rheumatology and Clinical Immunology, Charité Universitätsmedizin Berlin, corporate member of Freie Universität Berlin and Humboldt-Universität zu Berlin, Berlin, Germany

${ }^{8}$ Department of Neurology, Campus Benjamin Franklin, Charité -

Universitätsmedizin Berlin, corporate member of Freie Universität Berlin and Humboldt-Universität zu Berlin, Berlin, Germany
Acknowledgements We thank Miriam Rösner and Nadine Sylvester, who contributed to the translation of the study design into a practical protocol. Thanks to Wolfgang Keicher, AHG Ayurveda Handels GmbH, Hamburg and Seyfrieds Naturwaren, Osnabrück for the sponsoring of Pippali (Piper longum).We acknowledge support from the German Research Foundation (DFG) and the Open Access Publication Fund of Charité - Universitätsmedizin Berlin.

Contributors AM, CK, DS, TH and DAK-L designed the study and drafted the study protocol. AMH, MD, DAK-L, CK, MS and DS developed the interventional concept. TH and AM procured funding. NS and FIK performed statistical planning, sample size calculation and randomisation. AMH wrote the initial draft of the manuscript and coordinated the editing process. All other authors (MD, DS, CK, NS, MJ, JMF, MS, MAK, JGS, TH, AM and DAK-L) contributed equally with edits, comments and feedback. All authors read and approved the final manuscript.

Funding There are no financial or non-financial competing interests to declare in relation to this manuscript by any of the authors. The clinical trial itself is sponsored by Corona foundation (Corona Stiftung, Deutsches Stiftungszentrum Essen), grant number S199/10063/2016.

Disclaimer The funding body had no role in the design of the study or the collection, analysis, and interpretation of data or in writing the manuscript.

Competing interests None declared.

Patient consent for publication Not required.

Provenance and peer review Not commissioned; externally peer reviewed.

Supplemental material This content has been supplied by the author(s). It has not been vetted by BMJ Publishing Group Limited (BMJ) and may not have been peer-reviewed. Any opinions or recommendations discussed are solely those of the author(s) and are not endorsed by BMJ. BMJ disclaims all liability and responsibility arising from any reliance placed on the content. Where the content includes any translated material, BMJ does not warrant the accuracy and reliability of the translations (including but not limited to local regulations, clinical guidelines, terminology, drug names and drug dosages), and is not responsible for any error and/or omissions arising from translation and adaptation or otherwise.

Open access This is an open access article distributed in accordance with the Creative Commons Attribution Non Commercial (CC BY-NC 4.0) license, which permits others to distribute, remix, adapt, build upon this work non-commercially, and license their derivative works on different terms, provided the original work is properly cited, appropriate credit is given, any changes made indicated, and the use is non-commercial. See: http://creativecommons.org/licenses/by-nc/4.0/.

ORCID iD

Anika M Hartmann http://orcid.org/0000-0002-0135-9643

\section{REFERENCES}

1 Silman AJ, Pearson JE. Epidemiology and genetics of rheumatoid arthritis. Arthritis Res 2002;4:S265.

2 Kitas GD, Gabriel SE. Cardiovascular disease in rheumatoid arthritis: state of the art and future perspectives. Ann Rheum Dis 2011;70:8-14.

3 Smolen JS, Aletaha D. Rheumatoid arthritis therapy reappraisal: strategies, opportunities and challenges. Nat Rev Rheumatol 2015;11:276-89.

4 Smolen JS, Aletaha D, McInnes IB. Rheumatoid arthritis. The Lancet 2016;388:2023-38.

5 Smolen JS, Breedveld FC, Burmester GR, et al. Treating rheumatoid arthritis to target: 2014 update of the recommendations of an international Task force. Ann Rheum Dis 2016;75:3-15.

6 Fiehn C, Holle J, Iking-Konert C, et al. [S2e guideline: treatment of rheumatoid arthritis with disease-modifying drugs]. $Z$ Rheumatol 2018;77:35-53.

7 Rossi E, Noberasco C, Picchi M, et al. Complementary and integrative medicine to reduce adverse effects of anticancer therapy. J Altern Complement Med 2018;24:933-41.

8 Rao JK, Mihaliak K, Kroenke K, et al. Use of complementary therapies for arthritis among patients of rheumatologists. Ann Intern Med 1999;131:409.

9 Almuhareb AM, Alhawassi TM, Alghamdi AA, et al. Prevalence of complementary and alternative medicine use among rheumatoid arthritis patients in Saudi Arabia. Saudi Pharm J 2019;27:939-44.

10 McInnes IB, Schett G. The pathogenesis of rheumatoid arthritis. N Engl J Med Overseas Ed 2011;365:2205-19. 
11 Zmora N, Suez J, Elinav E. You are what you eat: diet, health and the gut microbiota. Nat Rev Gastroenterol Hepatol 2019;16:35-56.

12 Dehner C, Fine R, Kriegel MA. The microbiome in systemic autoimmune disease: mechanistic insights from recent studies. Curr Opin Rheumatol 2019;31:201-7.

13 Peltonen R, Kjeldsen-Kragh J, Haugen M, et al. Changes of faecal flora in rheumatoid arthritis during fasting and one-year vegetarian diet. Br J Rheumatol 1994;33:638-43.

14 Guerreiro CS, Calado Ângelo, Sousa J, et al. Diet, microbiota, and gut Permeability-The unknown triad in rheumatoid arthritis. Front Med 2018;5:349.

15 du Teil Espina M, Gabarrini G, Harmsen HJM, et al. Talk to your gut: the oral-gut microbiome axis and its immunomodulatory role in the etiology of rheumatoid arthritis. FEMS Microbiol Rev 2019;43:1-18.

16 Ruff WE, Greiling TM, Kriegel MA. Host-microbiota interactions in immune-mediated diseases. Nat Rev Microbiol 2020;18:521-38.

17 Horta-Baas G, Romero-Figueroa MDS, Montiel-Jarquín AJ, et al Intestinal dysbiosis and rheumatoid arthritis: a link between gut microbiota and the pathogenesis of rheumatoid arthritis. J Immunol Res 2017:2017:1-13.

18 Maeda Y, Kurakawa T, Umemoto E, et al. Dysbiosis contributes to arthritis development via activation of autoreactive T cells in the intestine. Arthritis \& Rheumatology 2016;68:2646-61.

19 Kriegel MA. Self or non-self? the multifaceted role of the microbiota in immune-mediated diseases. Clin Immunol 2015;159:119-21.

20 Horne BD, May HT, Anderson JL, et al. Usefulness of routine periodic fasting to lower risk of coronary artery disease in patients undergoing coronary angiography. Am J Cardiol 2008;102:814-9.

21 Göhler L, Hahnemann T, Michael N, et al. Reduction of plasma catecholamines in humans during clinically controlled severe underfeeding. Prev Med 2000;30:95-102.

22 Kjeldsen-Kragh J, Haugen M, Borchgrevink CF, Laerum E, et al. Controlled trial of fasting and one-year vegetarian diet in rheumatoid arthritis. Lancet 1991;338:899-902.

23 Maeda Y, Kurakawa T, Umemoto E, et al. Dysbiosis contributes to arthritis development via activation of autoreactive T cells in the intestine. Arthritis Rheumatol 2016;68:2646-61.

24 Scher JU, Littman DR, Abramson SB. Microbiome in inflammatory arthritis and human rheumatic diseases. Arthritis Rheumatol 2016;68:35-45.

25 Vaahtovuo J, Munukka E, Korkeamäki M, et al. Fecal microbiota in early rheumatoid arthritis. J Rheumatol 2008;35:1500-5.

26 De Luca F, Shoenfeld Y. The microbiome in autoimmune diseases. Clin Exp Immunol 2019;195:74-85.

27 Cheng Z, Do T, Mankia K, et al. Dysbiosis in the oral microbiomes of anti-CCP positive individuals at risk of developing rheumatoid arthritis. Ann Rheum Dis 2021;80:annrheumdis-2020-216972.

28 Masuko K. A Potential Benefit of "Balanced Diet" for Rheumatoid Arthritis. Front Med 2018;5:141.

29 Konig MF, Abusleme L, Reinholdt J, et al. Aggregatibacter actinomycetemcomitans-induced hypercitrullination links periodontal infection to autoimmunity in rheumatoid arthritis. Sci Trans/ Med 2016;8:369ra176-369.

30 Philippou E, Nikiphorou E. Are we really what we eat? nutrition and its role in the onset of rheumatoid arthritis. Autoimmun Rev 2018;17:1074-7.

31 Rinninella E, Cintoni M, Raoul P, et al. Gut microbiota during dietary restrictions: new insights in non-communicable diseases. Microorganisms 2020;8. doi:10.3390/microorganisms8081140. [Epub ahead of print: 2807 2020]

32 Schmidt NS, Lorentz A. Dietary restrictions modulate the gut microbiota: implications for health and disease. Nutr Res 2021;89:10-22.

33 de Cabo R, Mattson MP. Effects of intermittent fasting on health, aging, and disease. New England Journal of Medicine 2019;381:2541-51.

34 Mattson MP, Longo VD, Harvie M. Impact of intermittent fasting on health and disease processes. Ageing Res Rev 2017;39:46-58.

35 Mattson MP, Longo VD, Harvie M. Impact of intermittent fasting on health and disease processes. Ageing Res Rev 2017;39:46-58.

36 Fraser DA, Thoen J, Djøseland O, et al. Serum levels of interleukin-6 and dehydroepiandrosterone sulphate in response to either fasting or a ketogenic diet in rheumatoid arthritis patients. Clin Exp Rheumatol 2000;18:357-62.

37 Choi IY, Lee C, Longo VD. Nutrition and fasting mimicking diets in the prevention and treatment of autoimmune diseases and immunosenescence. Mol Cell Endocrinol 2017;455:4-12.

38 Longo VD, Mattson MP, Valter M. Fasting: molecular mechanisms and clinical applications. Cell Metab 2014;19:181-92.

39 Kjeldsen-Kragh J, Rashid T, Dybwad A, et al. Decrease in antiProteus mirabilis but not anti-Escherichia coli antibody levels in rheumatoid arthritis patients treated with fasting and a one year vegetarian diet. Ann Rheum Dis 1995;54:221-4.

40 Koppold-Liebscher D, Kessler CS, Steckhan N, et al. Short-Term fasting accompanying chemotherapy as a supportive therapy in gynecological cancer: protocol for a multicenter randomized controlled clinical trial. Trials 2020;21:854.

41 Hagen KB, Byfuglien MG, Falzon L, et al. Dietary interventions for rheumatoid arthritis. Cochrane Database Syst Rev 2009;February.

42 Alwarith J, Kahleova H, Rembert E, et al. Nutrition interventions in rheumatoid arthritis: the potential use of plant-based diets. A review. Front Nutr 2019;6:141.

43 Philippou E, Petersson SD, Rodomar C, et al. Rheumatoid arthritis and dietary interventions: systematic review of clinical trials. Nutr Rev 2021;79:410-28.

44 Khanna S, Jaiswal KS, Gupta B. Managing rheumatoid arthritis with dietary interventions. Front Nutr 2017;4:52.

45 Gioia C, Lucchino B, Tarsitano MG, et al. Dietary habits and nutrition in rheumatoid arthritis: can diet influence disease development and clinical manifestations? Nutrients 2020;12:1456.

46 Müller H, de Toledo FW, Resch KL, Müller FWDTK H. Fasting followed by vegetarian diet in patients with rheumatoid arthritis: a systematic review. Scand J Rheumatol 2001;30:1-10.

47 Michalsen A, Li C. Fasting therapy for treating and preventing disease - current state of evidence. Forsch Komplementmed 2013;20:444-53.

48 Sköldstam L, Larsson L, Lindström FD. Effect of fasting and lactovegetarian diet on rheumatoid arthritis. Scand J Rheumatol 1979;8:249-55.

49 Sköldstam L. Fasting and vegan diet in rheumatoid arthritis. Scand J Rheumatol 1986;15:219-21.

50 Kouda K, Iki M. Beneficial effects of mild stress (hormetic effects): dietary restriction and health. J Physiol Anthropol 2010;29:127-32.

51 Caughey GE, Mantzioris E, Gibson RA, et al. The effect on human tumor necrosis factor alpha and interleukin 1 beta production of diets enriched in n-3 fatty acids from vegetable oil or fish oil. Am J Clin Nutr 1996;63:116-22.

52 Adam O, Beringer C, Kless T, et al. Anti-Inflammatory effects of a low arachidonic acid diet and fish oil in patients with rheumatoid arthritis. Rheumatol Int 2003;23:27-36.

53 Chan A-W, Tetzlaff JM, Altman DG, et al. Spirit 2013 statement: defining standard protocol items for clinical trials. Ann Intern Med 2013;158:200-7.

54 Li C, Ostermann T, Hardt M, et al. Metabolic and psychological response to 7 -day fasting in obese patients with and without metabolic syndrome. Forsch Komplementmed 2013;20:413-20.

55 Wilhelmi de Toledo F, Buchinger A, Burggrabe $\mathrm{H}$, et al. Fasting therapy - an expert panel update of the 2002 consensus guidelines. Forsch Komplementmed 2013;20:434-43.

56 Stange R, Pflugbeil C, Michalsen A, et al. Therapeutic fasting in patients with metabolic syndrome and impaired insulin resistance. Forsch Komplementmed 2013;20:421-6.

57 Deutsche Gesellschaft für Ernährung e. V. Rheumadiät: Deutsche Gesellschaft für Ernährung E. V, 2008. Available: https://www. dge.de//wissenschaft/weitere-publikationen/fachinformationen/ rheumadiaet/ accessed 01.072021

58 Calder PC. Eicosanoids. Essays Biochem 2020;64:423-41.

59 Lewis RA, Austen KF, Soberman RJ. Leukotrienes and other products of the 5-lipoxygenase pathway. New England Journal of Medicine 1990;323:645-55.

$60 \mathrm{Li} \mathrm{X}, \mathrm{Bi} \mathrm{X}$, Wang S, et al. Therapeutic potential of $\omega-3$ polyunsaturated fatty acids in human autoimmune diseases. Front Immunol 2019;10:2241.

61 Innes JK, Calder PC. Omega-6 fatty acids and inflammation. Prostaglandins Leukot Essent Fatty Acids 2018;132:41-8.

62 Innis SM. Omega-3 fatty acid biochemistry: perspectives from human nutrition. Mil Med 2014;179:82-7.

63 Wilhelmi de Toledo F, Grundler F, Bergouignan A, et al. Safety, health improvement and well-being during a 4 to 21-day fasting period in an observational study including 1422 subjects. PLoS One 2019;14:e0209353.

64 Finnell JS, Saul BC, Goldhamer AC, et al. Is fasting safe? A chart review of adverse events during medically supervised, water-only fasting. BMC Complement Altern Med 2018;18:67.

65 Häupl T, Sörensen T, Boyer M. SAT0249 reduction of monocyte activation by bowel cleanse and one week fasting suggests permanent pathogenetic triggering from the gut in rheumatoid arthritis. Annals of the Rheumatic Diseases 2018;77:986-7.

66 Rincón J, Krook A, Galuska D, Rincón, Krook G, et al. Altered skeletal muscle glucose transport and blood lipid levels in habitual cigarette smokers. Clin Physiol 1999;19:135-42. 
67 Hiller K, Hangebrauk J, Jäger C, et al. MetaboliteDetector: comprehensive analysis tool for targeted and nontargeted GC/MS based metabolome analysis. Anal Chem 2009;81:3429-39.

68 Kozich JJ, Westcott SL, Baxter NT, et al. Development of a dualindex sequencing strategy and curation pipeline for analyzing amplicon sequence data on the MiSeq illumina sequencing platform. Appl Environ Microbiol 2013;79:5112-20.

69 Greiling TM, Dehner C, Chen X, et al. Commensal orthologs of the human autoantigen Ro60 as triggers of autoimmunity in lupus. Sci Transl Med 2018;10:eaan2306.

70 Ruff WE, Dehner C, Kim WJ, et al. Pathogenic autoreactive T and $B$ cells cross-react with mimotopes expressed by a common human gut commensal to trigger autoimmunity. Cell Host Microbe 2019;26:100-13.

71 Sv B. Flexible imputation of missing data. New York: CRC Press, Taylor and Francis Group, 2018.

72 Badsha $\mathrm{H}$. Role of diet in influencing rheumatoid arthritis disease activity. Open Rheumatol J 2018;12:19-28.

73 Bustamante MF, Agustín-Perez M, Cedola F, et al. Design of an antiinflammatory diet (ITIS diet) for patients with rheumatoid arthritis. Contemp Clin Trials Commun 2020;17:100524.

74 Forsyth C, Kouvari M, D'Cunha NM, et al. The effects of the Mediterranean diet on rheumatoid arthritis prevention and treatment: a systematic review of human prospective studies. Rheumatol Int 2018;38:737-47.

75 Vadell AKE, Bärebring L, Hulander E, et al. Anti-inflammatory Diet In Rheumatoid Arthritis (ADIRA)-a randomized, controlled crossover trial indicating effects on disease activity. Am J Clin Nutr 2020;111:1203-13.

76 Genel F, Kale M, Pavlovic N, et al. Health effects of a lowinflammatory diet in adults with arthritis: a systematic review and meta-analysis. J Nutr Sci 2020;9:e37.

77 Johansson K, Askling J, Alfredsson L, et al. Mediterranean diet and risk of rheumatoid arthritis: a population-based case-control study. Arthritis Res Ther 2018;20:175.

78 Nguyen Y, Salliot C, Gelot A, et al. Mediterranean diet and risk of rheumatoid arthritis: findings from the French E3N-EPIC cohort study. Arthritis Rheumatol 2021;73:69-77.

79 Smiljanovic B, Radzikowska A, Kuca-Warnawin E, et al. Monocyte alterations in rheumatoid arthritis are dominated by preterm release from bone marrow and prominent triggering in the joint. Ann Rheum Dis 2018;77:300-8.

80 Jordan S, Tung N, Casanova-Acebes M, et al. Dietary intake regulates the circulating inflammatory monocyte pool. Cell 2019;178:1102-14.

$81 \mathrm{Xu} \mathrm{H}$, Zhao H, Fan D, et al. Interactions between gut microbiota and immunomodulatory cells in rheumatoid arthritis. Mediators Inflamm 2020;2020:1430605.
82 Häger J, Bang H, Hagen M, et al. The role of dietary fiber in rheumatoid arthritis patients: a feasibility study. Nutrients 2019;11:2392.

83 Ang QY, Alexander M, Newman JC, et al. Ketogenic diets alter the gut microbiome resulting in decreased intestinal Th17 cells. Cell 2020;181:1263-75.

84 Zegarra-Ruiz DF, El Beidaq A, Iñiguez AJ, et al. A Diet-Sensitive commensal Lactobacillus strain mediates TLR7-Dependent systemic autoimmunity. Cell Host Microbe 2019;25:113-27.

85 Tajik N, Frech M, Schulz O, et al. Targeting zonulin and intestinal epithelial barrier function to prevent onset of arthritis. Nat Commun 2020;11:1995.

86 Bai Y, Li Y, Marion T, et al. Resistant starch intake alleviates collageninduced arthritis in mice by modulating gut microbiota and promoting concomitant propionate production. J Autoimmun 2021:116:102564.

87 Desai MS, Seekatz AM, Koropatkin NM, et al. A dietary FiberDeprived gut microbiota degrades the colonic mucus barrier and enhances pathogen susceptibility. Cell 2016;167:1339-53.

88 Zimmer J, Lange B, Frick J-S, et al. A vegan or vegetarian diet substantially alters the human colonic faecal microbiota. Eur $\mathrm{J}$ Clin Nutr 2012;66:53-60.

89 David LA, Maurice CF, Carmody RN, et al. Diet rapidly and reproducibly alters the human gut microbiome. Nature 2014;505:559-63.

90 Trefflich I, Jabakhanji A, Menzel J, et al. Is a vegan or a vegetarian diet associated with the microbiota composition in the gut? results of a new cross-sectional study and systematic review. Crit Rev Food Sci Nutr 2020;60:2990-3004.

91 Kolodziejczyk AA, Zheng D, Elinav E. Diet-microbiota interactions and personalized nutrition. Nat Rev Microbiol 2019;17:742-53.

92 Johnson AJ, Zheng JJ, Kang JW, et al. A guide to Diet-Microbiome study design. Front Nutr 2020;7:79.

93 Hafström I, Ringertz B, Spångberg A, et al. A vegan diet free of gluten improves the signs and symptoms of rheumatoid arthritis: the effects on arthritis correlate with a reduction in antibodies to food antigens. Rheumatology 2001;40:1175-9.

94 McDougall J, Bruce B, Spiller G, et al. Effects of a very low-fat, vegan diet in subjects with rheumatoid arthritis. J Altern Complement Med 2002;8:71-5.

95 Willett W, Rockström J, Loken B, et al. Food in the Anthropocene: the EAT-Lancet Commission on healthy diets from sustainable food systems. The Lancet 2019:393:447-92.

96 Wasmann KA, Wijsman P, van Dieren S, et al. Partially randomised patient preference trials as an alternative design to randomised controlled trials: systematic review and meta-analyses. BMJ Open 2019;9:e031151.

97 Williams JB, Popp D, Kobak KA, et al. P-640 - The power of expectation bias. European Psychiatry 2012;27:1. 